\title{
Formal comparison of SUSY in the nuclear U(6/2) model and in quantum field theory
}

\author{
R. V. Jolos ${ }^{1,2}$ and P. von Brentano ${ }^{1}$, \\ ${ }^{1}$ Institut für Kernphysik, Universität zu Köln, Köln, 50937 Köln, Germany \\ ${ }^{2}$ Bogoliubov Laboratory of Theoretical Physics, Joint Institute for Nuclear Research, 141980 \\ Dubna, Russia
}

(November 10, 2018)

\begin{abstract}
A nuclear physics example of the $\mathrm{U}(6 / 2)$ supersymmetry group is considered. It is shown that this group contains a supersymmetric subgroup with a structure similar to the SUSY model of the quantum field theory (QFT). A comparison of two models helps to clarify the relation between the supersymmetry schemes of QFT and of nuclear physics. Using this similarity relation between the numbers of the bosonic and fermionic states similar to the fundamental relation in QFT is obtained. For those supermultiplets with at least two fermions the numbers of the bosonic and fermionic states are equal as in QFT.
\end{abstract}

\section{INTRODUCTION}

Supersymmetry is a very interesting subject which is widely discussed in different subfields of physics. Supersymmetry has a long and interesting history. It was discovered in the particle physics, however, the ideas of supersymmetry have found in nuclear structure physics a very extensive application. Several good examples of an approximate dynamical supersymmetry have been found there. The investigations of the dynamical supersymmetries in nuclear structure physics has been initiated by F.Iachello and the results obtained are summarized in [四].

It was found that the combined algebras are particularly useful in a description of the properties of odd-even nuclei. Moreover, an attempt to get a classification in which several nuclei including even and odd ones are described by the same Hamiltonian has been realised and very interesting results have been obtained [1 13]

However, the formalism to introduce the supersymmetries used is different in particle physics 114 16] and in nuclear structure physics. This makes a comparison more difficult and also restricts the application of the examples found in nuclear physics. In nuclear structure theory the main attention has been paid to the construction of the Hamiltonians, which exhibit dynamical supersymmetries. Such Hamiltonians are represented by the sums of the Casimir operators for an appropriate subgroup chain of $U(6 / \Omega)$, where $\Omega$ is a maximum number of the fermions in a system. Taken with the same parameters the Hamiltonians constructed in this way have been applied to the description of different nuclei including 
even and odd ones. However, previous works considered those subgroup chains in which already at the first step a classical Lie algebra formed by the operators of the Bose sector of $\mathrm{U}(6 / \Omega)$ appears.

For this reason, the operators of the Fermi sector (in the following called super operators), i.e. the operators transforming bosons into fermions and vice versa [17], do not appear explicitly in the subgroup chains. The super operators of the basic $\mathrm{U}(6 / \Omega)$ graded algebra commute with a Hamiltonian which is a function of only the Casimir operator of $U(6 / \Omega)$, which is the operator of the total number of bosons plus fermions. Such a Hamiltonian is too simple to describe real nuclei and it does not contain an interaction. In other words, the super operators analogous to those which are included in a construction of the Lagrangians in a QFT do not appear in this consideration. This indicates the difference in the schemes of the realization of the supersymmetry ideas in nuclear and particle physics. The examples of the dynamical supersymmetries in nuclear structure physics where the super operators have been explicitly introduced and discussed are given in [18,19]. However, in these examples the structure of supergroups is different from that of QFT.

In this paper we consider a well known example of the supersymmetry from the Interacting Boson-Fermion model, which on one side has a good experimental realization in ${ }^{102} \mathrm{Ru}$, ${ }^{103} \mathrm{Rh}$ and ${ }^{104} \mathrm{Pd}$ [1,9] and on the other side has an algebraic structure which is similar to the SUSY model in QFT, however. It is the main aim of the present paper to demonstrate this similarity and consider the consequences of it.

\section{U(6/2) NUCLEAR STRUCTURE MODEL}

Below we shall assume that fermions occupy a single particle state with angular momentum $j=1 / 2$ (e.g. a $p_{1 / 2}$ state). Thus, the underlying graded algebra is $U(6 / 2)$.

Before starting the consideration let us introduce a Hamiltonian which has a simpler structure and which can serve as an introduction to the more general case considered below.

The simplest Hamiltonian for a system of the noninteracting monopole $s$-bosons and a fermion with a spin equal $\frac{1}{2}$ and a good helicity quantum number is

$$
H=\epsilon\left(a_{\frac{1}{2} \frac{1}{2}}^{+} a_{\frac{1}{2} \frac{1}{2}}+s^{+} s\right)
$$

This is a standard example found in textbooks [20], by which many features of the supersymmetries can be illustrated. However, this Hamiltonian does not possess rotational symmetry and is not realized in nuclear physics. A simple extension of this Hamiltonian which possesses rotational symmetry is

$$
H=\epsilon\left(a_{\frac{1}{2} \frac{1}{2}}^{+} a_{\frac{1}{2} \frac{1}{2}}+a_{\frac{1}{2}-\frac{1}{2}}^{+} a_{\frac{1}{2}-\frac{1}{2}}+s^{+} s\right) .
$$

The super operators commuting with the Hamiltonian (2) are $a_{\frac{1}{2} \frac{1}{2}}^{+} s$ and $a_{\frac{1}{2}-\frac{1}{2}}^{+} s$. They transform eigenstates of this Hamiltonian into another ones with the same energy by changing the number of fermions in the state.

We go one step further and introduce the Hamiltonian which is used in the nuclear physics example below and includes not only monopole $s$-bosons but also quadrupole $d$-bosons. We shall consider the case of the so called $U(5)$ dynamical symmetry limit, for which the numbers of $s-$ and $d$-bosons are conserved separately. In this case the Hamiltonian takes the form 


$$
H=\epsilon\left(N_{F}+N_{s}\right)+H_{d}
$$

where $N_{s} \equiv s^{+} s$ and $N_{F} \equiv \sum_{m} a_{1 / 2 m}^{+} a_{1 / 2 m}$ are the number operators for the $s$-bosons and the fermions, correspondingly, and

$$
H_{d}=\epsilon_{d} \sum_{\mu} d_{\mu}^{+} d_{\mu}+\sum_{L=0,2,4} C_{L}\left(d^{+} d^{+}\right)_{L M}(d d)_{L M}
$$

The supercharges commuting with the Hamiltonian (3) are

$$
\begin{gathered}
P_{1 / 2 m}=a_{1 / 2 m}^{+} s, \quad P_{1 / 2 m}^{+}=s^{+} a_{1 / 2 m}, \\
{\left[H, P_{1 / 2 m}\right]=\left[H, P_{1 / 2 m}^{+}\right]=0}
\end{gathered}
$$

Let us consider a graded algebra which contains $P_{1 / 2 m}$ and $P_{1 / 2 m}^{+}$operators. The anticommutator of the supercharge operators $P_{1 / 2 m}$ and $P_{1 / 2 m}^{+}$is

$$
\left\{P_{1 / 2 m}, P_{1 / 2 m^{\prime}}^{+}\right\}=\delta_{m m^{\prime}}\left(N_{s}+\frac{1}{2} N_{F}\right)-\sqrt{2}(-1)^{1 / 2+m^{\prime}} C_{1 / 2 m 1 / 2-m^{\prime}}^{1 \eta} S_{1 \eta},
$$

where $S_{1 \eta}$ is the operator of the spin of the fermions

$$
S_{1 \eta}=\frac{\sqrt{3}}{2} \sum_{m, m^{\prime}} C_{1 / 2 m 1 \eta}^{1 / 2 m^{\prime}} a_{1 / 2 m^{\prime}}^{+} a_{1 / 2 m}
$$

and $C_{b \beta c \gamma}^{a \alpha}$ is a Clebsch-Gordan coefficient. The next set of the anticommutators is simple

$$
\left\{P_{1 / 2 m}^{+}, P_{1 / 2 m^{\prime}}^{+}\right\}=\left\{P_{1 / 2 m}, P_{1 / 2 m^{\prime}}\right\}=0
$$

The operators $N_{s}$ and $N_{F}$ commute with each other and with $S_{1 \eta}$. The operators $S_{1 \eta}$ satisfy the usual commutation relations for spin algebra

$$
\left[S_{1 \eta}, S_{1 \eta^{\prime}}\right]=\sqrt{2} C_{1 \eta^{\prime} 1 \eta^{\prime \prime}}^{1 \eta^{\prime \prime}} S_{1 \eta^{\prime \prime}}
$$

Finally we consider the commutation relations of the operators $N_{s}, N_{F}$ and $S_{1 \eta}$ with the supercharges $P_{1 / 2 m}$ and $P_{1 / 2 m}^{+}$. They are

$$
\begin{gathered}
{\left[P_{1 / 2 m}, N_{s}\right]=P_{1 / 2 m},} \\
{\left[P_{1 / 2 m}, N_{F}\right]=-P_{1 / 2 m},} \\
{\left[P_{1 / 2 m}, S_{1 \eta}\right]=\frac{\sqrt{3}}{2} C_{1 / 2 m 1 \eta}^{1 / 2 m^{\prime}} P_{1 / 2 m^{\prime}}}
\end{gathered}
$$

The operators $P_{1 / 2 m}, P_{1 / 2 m}^{+}, N_{s}, N_{F}$ and $S_{1 \eta}$ form a graded algebra, which is $U(1 / 2)$, and the Hamiltonian (3) corresponds to the following reduction chain 


$$
U(6 / 2) \supset U(5) \otimes U(1 / 2)
$$

The multiplets of the eigenstates of the Hamiltonian (31) with the same energy combine the states with different number of fermions. For this reason they are called supermultiplets. Applying the super operators $P_{1 / 2 m}, P_{1 / 2 m}^{+}$we can transform one member of the supermultiplet into an other one with different number of fermions. All states belonging to the supermultiplet can be created by a repeated application of the supersymmetric operators $P_{\frac{1}{2} \frac{1}{2}}$ and $P_{\frac{1}{2}-\frac{1}{2}}$ to some basic state $\mid n_{s}, n_{d}>$ with $n_{s}$ and $n_{d}$ being the numbers of the $s_{-}^{-}$ and $d$ - bosons in this state, correspondingly. This state does not contain fermions. The complete supermultiplet thus includes the following states

$$
\begin{array}{r}
\left|n_{s}, n_{d}>\equiv\left(s^{+}\right)^{n_{s}}\left\{\left(d^{+}\right)^{n_{d}}\right\}_{I M}\right| 0> \\
P_{\frac{1}{2} \frac{1}{2}}\left|n_{s}, n_{d}>, P_{\frac{1}{2}-\frac{1}{2}}\right| n_{s}, n_{d}> \\
P_{\frac{1}{2} \frac{1}{2}} P_{\frac{1}{2}-\frac{1}{2}} \mid n_{s}, n_{d}>.
\end{array}
$$

Above the state $\mid n_{s}, n_{d}>$ is an analog of the Clifford (fermion) vacuum of the Susy model of the QFT. Examples of the complete supermultiplets are shown in Table 1. Looking at these examples we see that there is an interesting relation between the total number of the bosonic states, which include by definition states without fermions and also states with even numbers of fermions, and fermionic states, i.e. states with odd numbers of fermions, in the supermultiplet. In the supermultiplets, which include the states with the number of $s$-bosons equal or exceeding two, the total number of the fermionic (magnetic) substates is equal to the number of the bosonic (magnetic) substates. In the supermultiplets, which include states with the number of $s$-bosons equal or not exceeding one, the total number of the fermionic (magnetic) substates is equal to twice the number of the bosonic (magnetic) substates. This relation will be extended in the next section where the formal proof will be presented.

A nice and well known example of the approximate supersymmetry in nuclear physics corresponding to the Hamiltonian (31) is given in [1.9]. It is a complete supermultiplet containing the three nuclei ${ }_{44}^{102} \mathrm{Ru}_{58}\left(N_{s}+N_{d}=7\right),{ }_{45}^{103} \mathrm{Rh}_{58}\left(N_{s}+N_{d}=6, N_{F}=1\right)$ and also the two quasiparticle states of ${ }_{46}^{104} \mathrm{Pd}_{58}\left(N_{s}+N_{d}=5, N_{F}=2\right)$. However, the experimental information on the two quasiparticle states in ${ }^{104} \mathrm{Pd}$ is insufficient. A possible candidate for the lowest state in ${ }^{104} \mathrm{Pd}$ belonging to the supermultiplet is $0^{+}(1793 \mathrm{KeV})$ state seen in $\beta^{-}$decay of ${ }^{104} \mathrm{Rh}$ with $\log f t=5.5$. Experiments on ${ }^{103} \mathrm{Rh}\left({ }^{3} \mathrm{He}, \mathrm{d}\right)$ will be important to clarify a situation. The odd proton occupies the $p_{1 / 2}$ single particle state. The experimental spectra are given in Fig.1. We put in Fig.1 the $0^{+}$two quasiparticle state of ${ }^{104} \mathrm{Pd}$ at the same energy as the ground states of ${ }^{102} \mathrm{Ru}$ and ${ }^{103} \mathrm{Rh}$ because in SUSY approach to nuclear structure we are dealing with the energies of the states relative to the lowest state of the multiplet in the same nucleus. This is also the reason why in (3) the same coefficient is used in front of $N_{F}$ and $N_{s}$ terms. The corresponding full Hamiltonian $\bar{H}$ which includes total energies is

$$
\bar{H}=\epsilon_{F} N_{F}+\epsilon_{s} N_{s}+H_{d} .
$$

If we consider only relative energies $\bar{H}$ is equivalent to $H$ of (3), however. This can be seen by rewriting $\bar{H}$ as follows 


$$
\bar{H}=\left(\epsilon_{F}-\epsilon_{s}\right) N_{F}+\epsilon_{s}\left(N_{F}+N_{s}\right)+H_{d} .
$$

One notes that the first term on the r.h.s. of (17) does not influence the relative energies. This is why this term is omitted which leads to the Hamiltonian $H$ of (3).

We see in Fig.1 that although the supersymmetry is realized with a good accuracy in ${ }^{102} \mathrm{Ru}$ and ${ }^{103} \mathrm{Rh}$ the symmetry is broken. So, to describe the experimental data in more details it is necessary to add to the Hamiltonian (33) a term which breaks the supersymmetry described above. This term can be choosen as

$$
H_{\text {break }}=\alpha N_{s} \cdot N_{F}+\beta \overrightarrow{L_{d}} \cdot \vec{S},
$$

where $\overrightarrow{L_{d}}=\sqrt{10}\left(d^{+} \tilde{d}\right)_{1}$ is the operator of the orbital momentum of $d$-bosons.

\section{FORMAL COMPARISON OF SUPERSYMMETRY IN U(6/2) MODEL AND IN QUANTUM FIELD THEORY}

In this section we shall compare the commutation and anticommutation relations between the operators of the $\mathrm{U}(1 / 2)$ superalgebra considered in the preceeding section with those of the SUSY model of the quantum field theory. This comparison will demonstrate a close similarity between both sets of relations and also some difference between them. The similarity will be used further to derive a formal relation between the numbers of the bosonic and fermionic states in the supermultiplets. In QFT the supercharges analogous to our operators $P_{1 / 2 m}$ and $P_{1 / 2 m}^{+}$are denoted by $Q_{\alpha}^{L}$ and $\left(Q_{\alpha}^{L}\right)^{+}$, where $\alpha$ is a spinorial index and $L$ is an index connected with an intrinsic symmetry group. They commute with the 4-dimensional Lorentz momentum $P_{\mu}$

$$
\left[Q_{\alpha}^{L}, P_{\mu}\right]=\left[\left(Q_{\alpha}^{L}\right)^{+}, P_{\mu}\right]=0,
$$

and therefore with the Hamiltonian, which is a $\mu=0$ component of $P_{\mu}$.

The anticommutator of the supercharge operators $Q_{\alpha}^{L}$ and $\left(Q_{\alpha}^{L}\right)^{+}$is

$$
\left\{Q_{\alpha}^{L},\left(Q_{\beta}^{M}\right)^{+}\right\}=2 \delta^{L M} \sum_{\mu} \sigma_{\alpha \beta}^{\mu} P_{\mu},
$$

where $\sigma^{\mu}$ is a Dirac matrix. The second anticommutator is

$$
\left\{Q_{\alpha}^{L}, Q_{\beta}^{M}\right\}=0 .
$$

In addition to the super operators $Q_{\alpha}^{L},\left(Q_{\alpha}^{L}\right)^{+}$and the Lorentz 4-dimensional momentum $P_{\mu}$ the SUSY model of QFT can includes also the boson type operators, which form the intrinsic symmetry Lie algebra in QFT, where they are denoted by $B_{l}$. The operators $B_{l}$ satisfy to the following commutation relations.

$$
\left[B_{l}, B_{m}\right]=i \sum_{k} f_{l m k} B_{k},
$$

where $f_{l m k}$ are the structure constants. The commutators between the intrinsic operators and super operators are 


$$
\left[Q_{\alpha}^{L}, B_{l}\right]=i \sum_{M} A_{l}^{L M} Q_{\alpha}^{M}
$$

Now these relations can be putted in a correspondence with those relations obtained in the preceeding section. This is done below, where the corresponding relations are connected by the left-right arrows

$$
\begin{gathered}
P_{1 / 2 m} \Longleftrightarrow Q_{\alpha}^{L}, H \Longleftrightarrow P_{\mu} \\
N_{s}-N_{F}, S_{1 \eta} \Longleftrightarrow B_{l}, \\
{\left[P_{1 / 2 m}, H\right]=0 \Longleftrightarrow\left[Q_{\alpha}^{L}, P_{\mu}\right]=0,} \\
\left\{P_{1 / 2 m^{\prime}}, P_{1 / 2 m}^{+}\right\}=\delta_{m m^{\prime}}\left(N_{s}+\frac{1}{2} N_{F}\right)+\sqrt{3} \sum_{\eta} C_{1 / 2 m 1 \eta}^{1 / 2 m^{\prime}} S_{1 \eta} \\
\left\{P_{1 / 2 m}, P_{1 / 2 m^{\prime}}\right\}=0 \Longleftrightarrow\left\{Q_{\alpha}^{L},\left(Q_{\beta}^{M}\right)^{+}=2 \delta^{L M} \sum_{\mu} \sigma_{\alpha \beta}^{\mu} P_{\mu}\right. \\
\left.\left[S_{1 \eta}, S_{1 \eta^{\prime}}\right]=\sqrt{2} C_{1 \eta^{\prime} 1 \eta^{\prime \prime}}^{1 \eta_{1 \eta^{\prime \prime}}} \Longleftrightarrow Q_{\beta}^{M}\right\}=0, \\
\left.\left[P_{1 / 2 m}, N_{s}\right]=P_{1 / 2 m},\left[P_{1 / 2 m}, N_{F}\right]=-B_{m}\right]=i \sum_{k} f_{l m k} B_{k} \\
\left.\sqrt{3} P_{1 / 2 m}, S_{1 \eta}\right]=-\frac{2 m}{2} C_{1 / 2 m 1 \eta} P_{1 / 2 m^{\prime}} \Longleftrightarrow\left[Q_{\alpha}^{L}, B_{l}\right]=i \sum_{M} A_{l}^{L M} Q_{\alpha}^{M}
\end{gathered}
$$

It is easy to see a strong similarity between analogous relations in the QFT and in the nuclear case. This similarity is evident for the relations (26,28 30). We notice, however, some difference in the structure of the expressions on the right hand sides of the anticommutators in (27). This distinction is due to the difference in the underlying bosonic algebras in QFT and in nuclear case. This difference is reflected in the relation between the numbers of the bosonic and fermionic states in the supermultiplets, which we shall consider below.

As in quantum field theory this relation can be obtained by multiplying both sides of the relation (7) by $(-1)^{N_{F}}$ and taking a trace. On the left side we get

$$
\begin{gathered}
\operatorname{Tr}\left((-1)^{N_{F}} P_{1 / 2 m} P_{1 / 2 m^{\prime}}^{+}+(-1)^{N_{F}} P_{1 / 2 m^{\prime}}^{+} P_{1 / 2 m}\right)= \\
\operatorname{Tr}\left((-1)^{N_{F}} P_{1 / 2 m} P_{1 / 2 m^{\prime}}^{+}+P_{1 / 2 m}(-1)^{N_{F}} P_{1 / 2 m^{\prime}}^{+}\right)= \\
\operatorname{Tr}\left((-1)^{N_{F}} P_{1 / 2 m} P_{1 / 2 m^{\prime}}^{+}-(-1)^{N_{F}} P_{1 / 2 m} P_{1 / 2 m^{\prime}}^{+}\right)=0
\end{gathered}
$$

Above we have used the invariance of the trace operation under the cyclical permutations of the operators and the commutation relation (12). Since the relation (7) can be also written as 


$$
\left\{P_{1 / 2 m}, P_{1 / 2 m^{\prime}}^{+}\right\}=\delta_{m m^{\prime}} s^{+} s+a_{1 / 2 m}^{+} a_{1 / 2 m^{\prime}}
$$

by taking $m=m^{\prime}$ and summing over $m$ we get

$$
\operatorname{Tr}\left((-1)^{\hat{N}_{F}}\left(2 \hat{N}_{s}+\hat{N}_{F}\right)\right)=0 .
$$

In fact (33) contains the relation between the numbers of the bosonic and fermionic states in the supermultiplets. To show it let us rewrite the trace in (33) in an explicit way as a sum of the diagonal matrix elements of the operator in circular brackets. Since the super operators on the left of (32) have nonzero matrix elements only between the states belonging to the same supermultiplet this sum is restricted to these states

$$
\sum_{a \in \text { supermultiplet }}<a\left|(-1)^{\hat{N}_{F}}\left(2 \hat{N}_{s}+\hat{N}_{F}\right)\right| a>=0
$$

Above the index $a$ includes bosonic states, i.e. the states without fermions and with two fermions, and fermionic states, i.e. states with one fermion. Since two fermions can be coupled in our case only to zero angular momentum the number of magnetic substates without fermions is equal to the number of magnetic substates with two fermions. Therefore, both are equal to the half of the total number of the bosonic magnetic substates in the supermultiplet, which we denote as $n_{B}$. Let $n_{F}$ be the total number of the fermionic states in the supermultiplet. Applying (34) to the supermultiplets with $N_{s}+N_{F} \equiv N_{s}^{\max } \geq 2$ one obtains

$$
\begin{array}{r}
\frac{1}{2} n_{B} \cdot 2 N_{s}^{\max }-n_{F}\left(2\left(N_{s}^{\max }-1\right)+1\right)+\frac{1}{2} n_{B}\left(2\left(N_{s}^{\max }-2\right)+2\right) \\
=\left(n_{B}-n_{F}\right)\left(2 N_{s}^{\max }-1\right)=0
\end{array}
$$

and thus $n_{B}=n_{F}$. Above $N_{s}^{\max }$ is a maximum possible number of the $s$-bosons in the states belonging to a supermultiplet.

For the supermultiplet with $N_{s}+N_{F}=1$ we get from (34)

$$
\frac{1}{2} n_{B} \cdot 1-n_{F}=0
$$

Thus we can formulate the following rules:

-In the supermultiplets which include the states with $N_{s}+N_{F} \geq 2$ the number of bosonic (magnetic) substates is equal to the number of the fermionic (magnetic) substates.

-In the supermultiplets containing states with $N_{s}+N_{F}=1$ the number of the fermionic (magnetic) substates is equal to twice the number of the bosonic (magnetic) substates.

There is some difference in the relations between the numbers of bosonic and fermionic states in nuclear case and in QFT where for all supermultiplets (excluding ground state) $n_{F}=n_{B}$. This difference is a consequence of the difference in the anticommutation relations (7) and (20).

Since the relation between the numbers of the bosonic and fermionic (magnetic) substates is a fundamental point in QFT, consider from this point of view the eigenstates of the Hamiltonians (11.2). The supermultiplets of the eigenstates of the Hamiltonian (10) correspond to the supermultiplets of the QFT with the zero rest mass Clifford (fermion) vacuum. In 
this case helicity is a good quantum number. The one-fermion state is, for instance, an analog of neutrino and a one $s$-boson state is an analog of $\mathrm{S}$-neutrino. In the case of the Hamiltonian (2) a one-fermion state is an analog of electron with two possible projections of the spin.

In the QFT the following expression has been derived for the Hamiltonian. If we multiply both sides of (20) by $\sigma_{\alpha \beta}^{0}$ and sum over $\alpha$ and $\beta$ we get

$$
H \equiv P_{0}=\frac{1}{4} \sum_{\alpha, L}\left\{Q_{\alpha}^{L},\left(Q_{\alpha}^{L}\right)^{+}\right\}
$$

In our case the relation, which can be derived in a similar way, is different because of the difference in the anticommutation relations (7) and (20)

$$
H=\frac{1}{2} N_{F}+\frac{1}{2} \sum_{m}\left\{P_{1 / 2 m}, P_{1 / 2 m}^{+}\right\}+H_{d}
$$

which is equivalent from the excitation energy point of view to

$$
H=\frac{1}{2} \sum_{m}\left\{P_{1 / 2 m}, P_{1 / 2 m}^{+}\right\}+H_{d}
$$

However, the ground state (i.e. the state with zero numbers of fermions, $s$ - and $d$ - bosons) of our Hamiltonian has zero energy as in QFT.

\section{CONCLUSION}

We have considered the comparison of the supersymmetry concept in nuclear structure theory and in quantum field theory. The nuclear structure model based on the U(6/2) graded algebra is considered. It is shown that the $\mathrm{U}(6 / 2)$ algebra has a graded subalgebra whose generators commute with the model Hamiltonian. Thus, the existence of the supersymmetric operators transforming bosons into fermions and vice versa and commuting with the Hamiltonian is demonstrated. We have furthermore obtained explicit expressions of the wavefunctions of the various members of supermultiplets. For supermultiplets with $N_{F}+N_{s} \geq 2$ we find an equal number of boson and fermion type (magnetic) substates in the supermultiplet. This corresponds to the fundamental property of supermultiplets in QFT.

\section{ACKNOWLEDGMENTS}

The authors would like to express their gratitude to Profs. R.F.Casten, A.Gelberg, T.Otsuka, P. van Isacker and M.Zirnbauer for discussions. The work was supported in part by the DFG under the contract Br 799/8-2. One of the authors (R. V. J) is grateful to the Universität zu Köln for support. 


\section{REFERENCES}

[1] F. Iachello and P. Van Isacker, The Interacting Boson-Fermion Model, (Cambridge University Press, Cambridge, 1991).

[2] A. Frank and P. Van Isacker, Algebraic Methods in Molecular and Nuclear Physics, (John Wiley \& Sons, New York, 1994).

[3] F. Iachello, Phys. Rev. Lett. 44, 772 (1980).

[4] A. B. Balantekin, I. Bars and F. Iachello, Nucl. Phys. A370, 284 (1981).

[5] F. Iachello and S. Kuyucak, Ann. Phys. (N.Y.) 136, 19 (1981).

[6] R. Bijker and V.K.B. Kota, Ann. Phys. (N.Y.) 156, 110 (1984).

[7] R. Bijker and F. Iachello, Ann. Phys. (N.Y.) 161, 360 (1985).

[8] R. Bijker and V.K.B. Kota, Ann. Phys. (N.Y.) 187, 148 (1988).

[9] J. Vervier, Revista Nuovo Cimento 10, No. 9.

[10] J. Vervier, in "Nuclear Structure, Reactions and Symmetries", Dubrovnik, Yugoslavia, 1986, eds. R. A. Meyer and V. Paar, (World Scientific, 1986), v.1, p. 175.

[11] J. A. Cizewski, in "Nuclear Structure, Reactions and Symmetries", Dubrovnik, Yugoslavia, 1986, eds. R. A. Meyer and V. Paar, (World Scientific, 1986), v.1, p. 181.

[12] D. D. Warner, R. F. Casten, and A. Frank, Phys. Lett. B, 180, 207 (1986).

[13] J. Vervier, P. Van Isacker, J. Jolie, V. K. B. Kota, and R. Bijker, Phys. Rev. C 32, 1406 (1985).

[14] J. Wess and J. Bagger, Supersymmetry and supergravity, (Princeton Series in Physics, Princeton University Press, 1983).

[15] P. Fayet and S. Ferrara, Phys. Rep.C 32, 249 (1977)

[16] H. Kalka and G. Soff, Supersymmetrie, (Teubner, Stuttgart, 1997).

[17] R.V.Jolos, P. von Brentano, A.Gelberg, K.-H. Kim, and T.Otsuka, Phys. Lett. B, 430, 1 (1998).

[18] A. B. Balantekin, Ann. Phys. 164, 277 (1985).

[19] I. Morrison and P. D. Jarvis, Nucl.Phys. A 435, 461 (1985).

[20] M. Kaku, Quantum field theory, (Oxford University Press, New York, Oxford, 1993). 


\section{TABLES}

TABLE I. Wave functions of the members of supermultiplets with various numbers of $s-$ and $d$-bosons. $P_{1 / 2 m}=a_{1 / 2 m}^{+} s$.

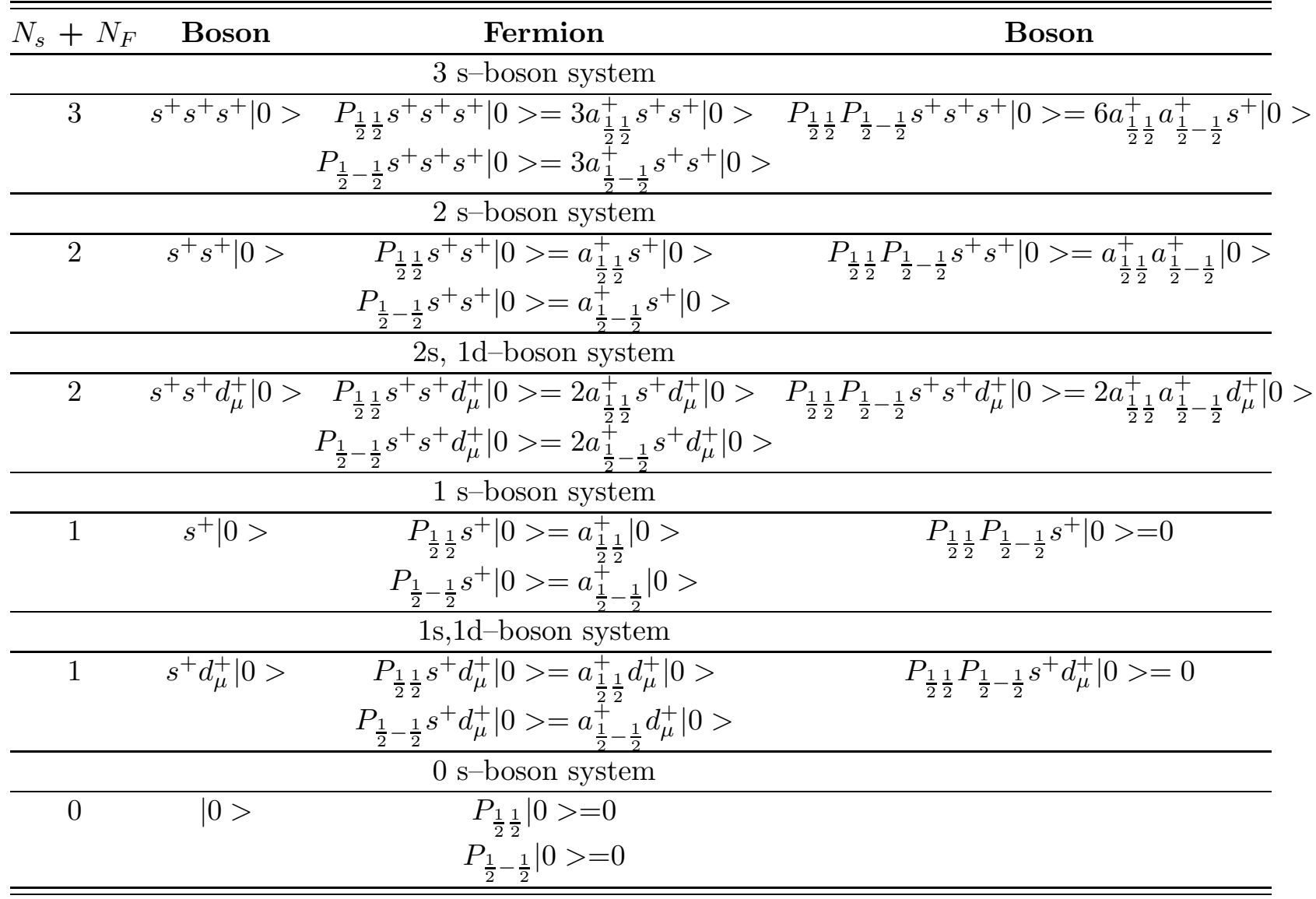




\section{FIGURES}

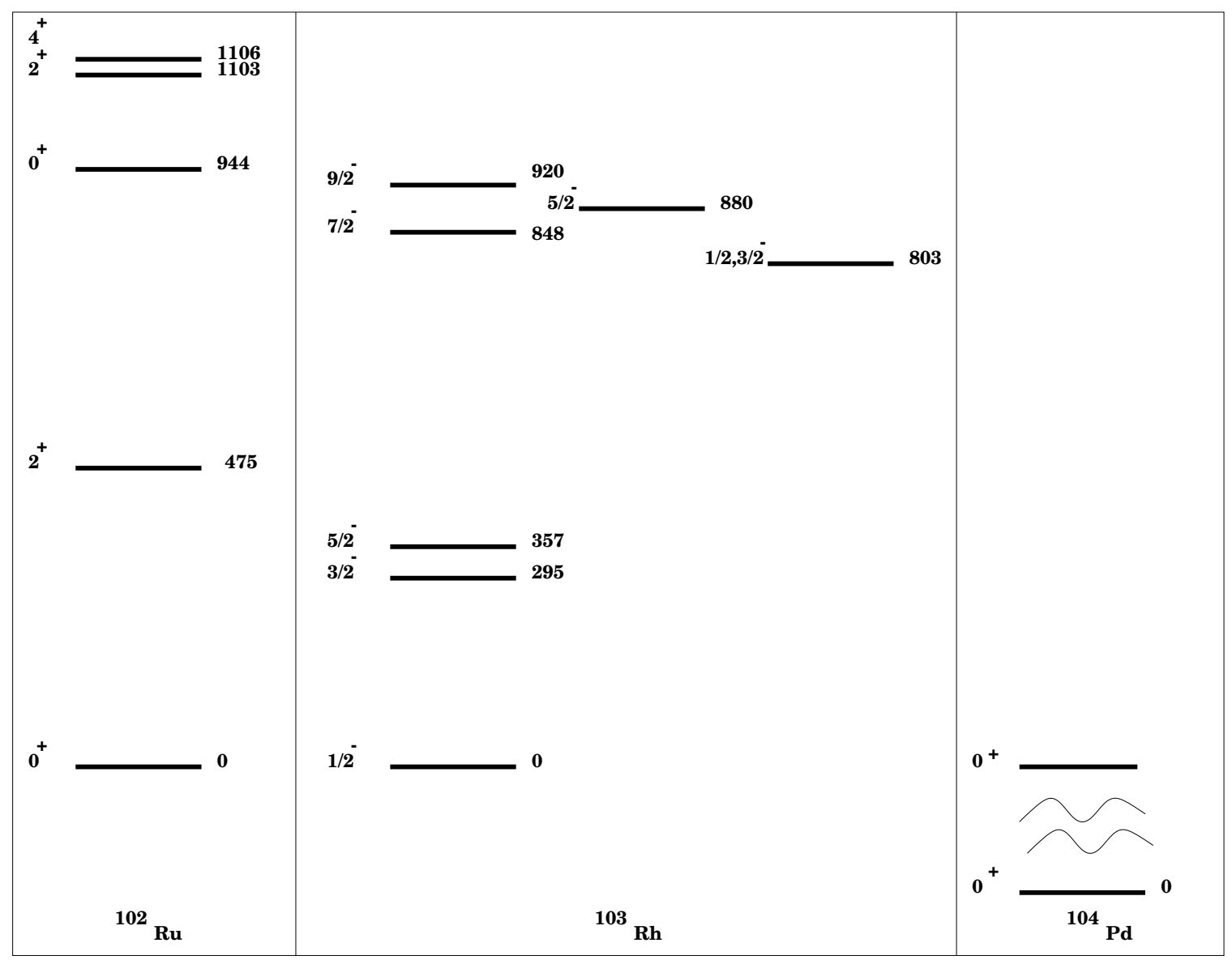

FIG. 1. An example of the $\mathrm{U}(6 / 2)$ supersymmetry in nuclei. A part of the experimental spectra of the ${ }^{102} \mathrm{Ru}^{-103}{ }^{10 h}{ }^{104} \mathrm{Pd}$ supermultiplet [1,9] is shown. The energies are given in $\mathrm{KeV}$. The two quasiparticle states in ${ }^{104} \mathrm{Pd}$ are not identified. A possible candidate for the lowest state in ${ }^{104} \mathrm{Pd}$ belonging to the supermultiplet is $0^{+}(1793 \mathrm{KeV})$ state seen in $\beta^{-}$decay of ${ }^{104} \mathrm{Rh}$ with $\log f t=5.5$. Experiments on ${ }^{103} \mathrm{Rh}\left({ }^{3} \mathrm{He}, \mathrm{d}\right)$ will be important to clarify a situation. We note that the number of boson type (magnetic) substates in the multiplet (in ${ }^{102} \mathrm{Ru}$ and ${ }^{104} \mathrm{Pd}$ ) is equal to the number of fermion type (magnetic) substates in the multiplet (in ${ }^{103} \mathrm{Rh}$ ). This holds for some low lying multiplets as e.g. the multiplets based on the $0^{+}$and $2^{+}$states in ${ }^{102} \mathrm{Ru}$. 